\title{
Magnetotransport in periodically modulated bilayer graphene
}

\author{
M. Zarenia, ${ }^{1, *}$ P. Vasilopoulos, ${ }^{2, \dagger}$ and F. M. Peeters ${ }^{1, \ddagger}$ \\ ${ }^{1}$ Department of Physics, University of Antwerp, Groenenborgerlaan 171, B-2020 Antwerp, Belgium \\ ${ }^{2}$ Department of Physics, Concordia University, 7141 Sherbrooke Ouest, Montréal, Québec, Canada H4B 1R6
}

(Received 12 March 2012; published 13 June 2012)

\begin{abstract}
Magnetotransport in bilayer graphene in the presence of a weak and periodic potential is investigated in the presence of a perpendicular magnetic field $B$. The modulation broadens the Landau levels into bands and for weak magnetic fields leads to the well-known Weiss oscillations in their bandwidth and their transport coefficients at very low $B$ and to the Shubnikov-de Haas oscillations at larger $B$. The amplitude of the Weiss oscillations is severely reduced if the periodic potentials applied to the two layers oscillate out of phase. We also contrast some results with those corresponding to single-layer graphene. Relative to them the flat-band condition and the oscillation amplitude differ substantially, due to the interlayer coupling, and agree only when this coupling is extremely weak. We further show that the Hall conductivity exhibits the well-known steps at half-integer and integer multiples of $4 e^{2} / h$ in single-layer and bilayer graphene, respectively, even for very weak magnetic fields. The results are pertinent to weak and periodic corrugations when the potential modulation dominates the strain-induced magnetic modulation.
\end{abstract}

DOI: 10.1103/PhysRevB.85.245426

PACS number(s): 71.10.Pm, 75.47.-m, 81.05.ue

\section{INTRODUCTION}

There exist many magnetotransport studies of the twodimensional electron gas (2DEG) subject to periodic potential $^{1,2}$ or magnetic modulations ${ }^{3,4}$ and to an external, mostly perpendicular to the 2DEG, magnetic field $B$ [see Ref. 2 for more references]. The interest results mainly from the fact that the modulation broadens the Landau levels (LLs) into bands and for weak magnetic fields leads to the well-known Weiss or commensurability oscillations in their bandwidth and their transport coefficients at very low $B$ and to the usual Shubnikov-de Haas oscillations at larger $B$. These Weiss oscillations are periodic in $1 / B$ and reflect the commensurability between the externally imposed period of the modulation and the diameter of the electron orbit at the Fermi level.

After the discovery of graphene $e^{5}$ similar studies appeared for single-layer graphene. 6,7 The qualitative behavior of the Weiss oscillations was found to be the same but an enhancement of their amplitude was predicted. ${ }^{6}$ These studies, however, were limited in number and were only partial. In fact, the longitudinal and transverse resistivities, as a function of $B$, oscillate in antiphase with each other. With the exception of Ref. 7 this aspect as well the oscillations in the Hall resistivity were not sufficiently addressed. Surprisingly, similar studies pertinent to bilayer graphene are, to our knowledge, absent.

Relatively recently single-layer graphene, subject to periodic corrugations, was studied in several papers. ${ }^{8,9}$ The corrugation induces a potential modulation as well as a magnetic modulation ${ }^{8}$ due to the stress. In these tight-binding treatments of corrugations almost invariably only the potential or the magnetic modulation was taken into account. Again, similar studies for bilayer graphene are, to our knowledge, absent.

In this paper we study magnetotransport in bilayer graphene in the presence of a weak and periodic potential modulation and of a perpendicular magnetic field $B$. Given the similarity between potential and magnetic modulations $s^{3,10}$ the results can be useful to periodic corrugations as well. Moreover, we critically contrast the results for single-layer and bilayer graphene and show that the well-known plateaus in the Hall resistivity for strong fields $B$ also occur for very weak $B$.

The paper is organized as follows. In Secs. II and III we present results for unmodulated and modulated bilayer graphene, respectively. Analytical and numerical results for the conductivities follow in Sec. IV and concluding remarks in Sec. V.

\section{UNMODULATED BILAYER GRAPHENE}

The one-particle Hamiltonian for bilayer graphene is given by $^{11}$

$$
H_{0}=\left(\begin{array}{cccc}
0 & \pi & t & 0 \\
\pi^{\dagger} & 0 & 0 & 0 \\
t & 0 & 0 & \pi^{\dagger} \\
0 & 0 & \pi & 0
\end{array}\right) .
$$

Here, $t \approx 400 \mathrm{meV}$ is the interlayer coupling, $B$ the external magnetic field perpendicular to the layers, $(\hbar / e B)^{1 / 2}=l_{B}$ the magnetic length, $v_{F}$ the Fermi velocity, and $\pi$ and $\pi^{\dagger}$ the momentum operators $\pi=-i \hbar v_{F}[d / d x+i d / d y-$ $(e B / \hbar) x], \pi^{\dagger}=-i \hbar v_{F}[d / d x-i d / d y+(e B / \hbar) x]$ written in the Landau gauge $\mathbf{A}=(0, B x, 0)$ for the vector potential $\mathbf{A}$. The eigenstates of Eq. (1) are four-component spinors,

$$
\Psi=\frac{1}{\sqrt{L_{y}}}\left(\begin{array}{c}
\varphi_{a}(x) \\
i \varphi_{b}(x) \\
\varphi_{c}(x) \\
i \varphi_{d}(x)
\end{array}\right) e^{i k_{y} y},
$$

with $k_{y}$ the wave vector. The solution of $H \psi=E \psi$ is given in Appendix $\mathrm{A}$ in terms of the dimensionless units $x \rightarrow l_{B} k_{y}+$ $x / l_{B}, \epsilon=\left(l_{B} / \hbar v_{F}\right) E, t \rightarrow\left(l_{B} / \hbar v_{F}\right) t$. The eigenvalues of the Hamiltonian (1) are

$$
\begin{aligned}
& \epsilon=s_{1}\left\{t^{2} / 2+2 n+1+s_{2} \sqrt{t^{4} / 4+(2 n+1) t^{2}+1}\right\}^{1 / 2}, \\
& n=0,1,2, \ldots
\end{aligned}
$$




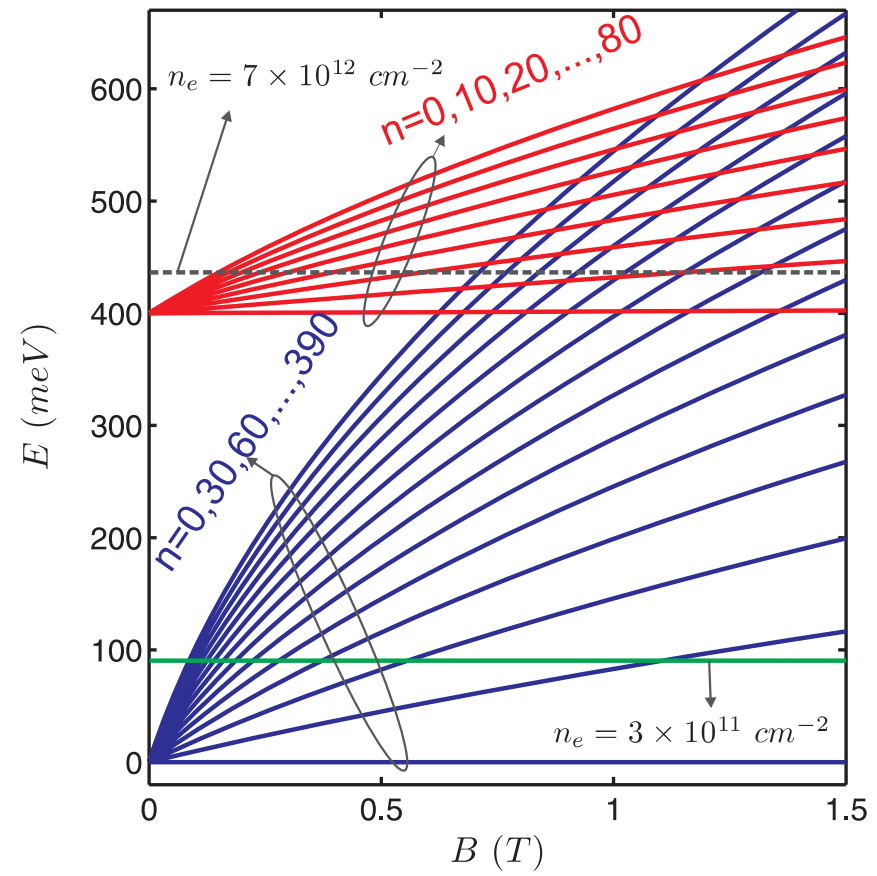

FIG. 1. (Color online) The energy spectrum of bilayer graphene as a function of an external magnetic field. The upper band is obtained for $s_{2}=1$ and the lower one for $s_{2}=-1$. The horizontal solid and dashed lines show the Fermi level $E_{F}$ with the corresponding values of the electron density $n_{e}$.

with $s_{1}= \pm 1$ and $s_{2}= \pm 1$ denoting the energy bands. For $s_{1}=1(-1)$ Eq. (3) gives the electron (hole) energy levels. If we set $t=0$ in Eq. (3) we obtain the energy levels in single-layer graphene. Notice that in obtaining Eq. (3) we neglected the Zeeman term because it is negligibly small in graphene, of the order of $0.5 \mathrm{meV}$ for $B=10 \mathrm{~T}$.

Some of the energy levels resulting from Eq. (3), with $s_{1}=1$, are plotted in Fig. 1 for weak magnetic fields $B$, $t=400 \mathrm{meV}$, and two values of the electron density $n_{e}$ as specified. The horizontal solid and dashed lines show the Fermi level $E_{F}$. As can be seen, the higher group of LLs $\left(s_{2}=1\right)$ is occupied for relatively high densities. Intentionally we chose a $n_{e}$ value that is about $20 \%$ higher than a recently reported experimental value. ${ }^{12}$

The corresponding eigenstates and their normalization are obtained in Appendix A [cf. Eqs. (A5)-(A14)], following the approach of Ref. 13. Rewritten here for convenience they read

$$
\begin{aligned}
& \varphi_{a}=N e^{-x^{2} / 2} H_{n}(x), \quad \varphi_{b}=-(2 n / \epsilon) N e^{-x^{2} / 2} H_{n-1}(x), \\
& \varphi_{c}=k_{n} N e^{-x^{2} / 2} H_{n}(x), \quad \varphi_{d}=\left(k_{n} / \epsilon\right) N e^{-x^{2} / 2} H_{n+1}(x),
\end{aligned}
$$

with $k_{n}=\left(\epsilon^{2}-2 n\right) / t \epsilon, H_{n}(x)$ the Hermite polynomial, and $N$ a normalization factor given by Eq. (A14).

\section{MODULATED BILAYER GRAPHENE}

In the presence of weak and periodic potentials $V_{1}(x)$ and $V_{2}(x)$ applied to the two layers, the total Hamiltonian becomes
$H=H_{0}+V$, where $V$ is the matrix

$$
V=\left(\begin{array}{cccc}
V_{1}(x) & 0 & 0 & 0 \\
0 & V_{1}(x) & 0 & 0 \\
0 & 0 & V_{2}(x) & 0 \\
0 & 0 & 0 & V_{2}(x)
\end{array}\right) .
$$

We consider three cases: (i) $V_{1}(x)=V_{2}(x)=V_{0} \cos (K x)$, (ii) $V_{1}(x)=V_{0} \cos (K x), \quad V_{2}(x)=0, \quad$ and (iii) $V_{1}(x)=$ $-V_{2}(x)=V_{0} \cos (K x), K=2 \pi / a$. These cases are schematically depicted at the top of Fig. 3. In all of them $V_{0} \ll E_{F}$ is the amplitude of the modulation and $a$ the period of the modulation. Case (iii) represents a system of kink-antikink potentials that results in an energy spectrum exhibiting chiral states. $^{14}$

Using the eigenfunctions of Eq. (1) and first-order perturbation theory we obtain

$$
\Delta E_{n, s, k_{y}}=V_{0} \cos \left(K x_{0}\right) e^{-u / 2} G_{n, s},
$$

where $u=K^{2} l_{B}^{2} / 2, x_{0}=l_{B}^{2} k_{y}$. For cases (i) and (iii), denoted below by $G_{n, s}^{+}$and $G_{n, s}^{-}$, respectively, we have

$$
\begin{aligned}
G_{n, s}^{ \pm}= & d_{n, s}^{2}\left\{\left(1 \pm k_{n, s}^{2}\right) L_{n}(u)+\left(2 n / \epsilon_{n, s}^{2}\right) L_{n-1}(u)\right. \\
& \left. \pm\left[2(n+1) k_{n, s}^{2} / \epsilon_{n, s}^{2}\right] L_{n+1}(u)\right\},
\end{aligned}
$$

with $s=\left\{s_{1}, s_{2}\right\}, \quad d_{n, s}=\left[k_{n, s}^{2}\left[1+2(n+1) / \epsilon_{n, s}^{2}\right]+1+2 n /\right.$ $\left.\epsilon_{n, s}^{2}\right]^{-1 / 2}$, and $L_{n}(u)$ are the Laguerre polynomials. For case (ii) we have the simpler result

$$
G_{n, s}=d_{n, s}^{2}\left\{L_{n}(u)+\left(2 n / \epsilon_{n, s}^{2}\right) L_{n-1}(u)\right\} .
$$

The result for single-layer graphene ${ }^{6}$ is obtained from Eq. (8) with the changes $d_{n, s}^{2} \rightarrow 1 / 2$ and $2 n / \epsilon_{n, s}^{2} \rightarrow 1$. The perturbed eigenvalues read

$$
E_{n, s, k_{y}}=E_{n, s}+\Delta E_{n, s, k_{y}},
$$

where $\Delta E_{n, s, k_{y}}$ is given by Eq. (6) with appropriate $G_{n, s}$ for cases (i)-(iii), and $E_{n, s}=\left(\hbar v_{F} / l_{B}\right) \epsilon_{n, s}$ with $\epsilon_{n, s}$ given by Eq. (3). Notice that Eqs. (7) and (8) are valid for $n \geqslant 1$, as well as for $n=0$, with $\epsilon \neq 0$. For $n=0$ and $\epsilon=0, G_{0, \pm,-}$ can be obtained using the wave spinors (B1) and (B2); the results are

$$
G_{0,+,-}=\left[2 L_{0}(u) \pm t^{2} L_{1}(u)\right] /\left(t^{2}+2\right), \quad G_{0,-,-}= \pm L_{0}(u),
$$

where the addition $(+)$ and subtraction $(-)$ signs correspond to cases (i) and (iii), respectively. For case (ii) we have $G_{0,+,-}=$ $2 L_{0}(u)$ and $G_{0,-,-}=0$.

The bandwidth (6) for case (i) is plotted in Fig. 2(a) versus (weak) field $B$ for the parameters shown in the figure. The dashed and solid curves correspond to single-layer and bilayer graphene. The large-amplitude oscillations are the Weiss or commensurability oscillations. The steplike structure, visible for $B \geqslant 0.2 \mathrm{~T}$, is due to the usual Shubnikov-de Haas oscillations. As can be seen, the single-layer bandwidth is larger than that of the bilayer; this depends mainly on the value of $t$. Notice that the minima and maxima of the two cases occur at different values of $B$. This is due to the fact that the flat-band condition $\left(\Delta E_{n, s, k_{y}}=0\right)$ differs substantially between the two cases [see Eq. (7) above and the text that follows]. This is 

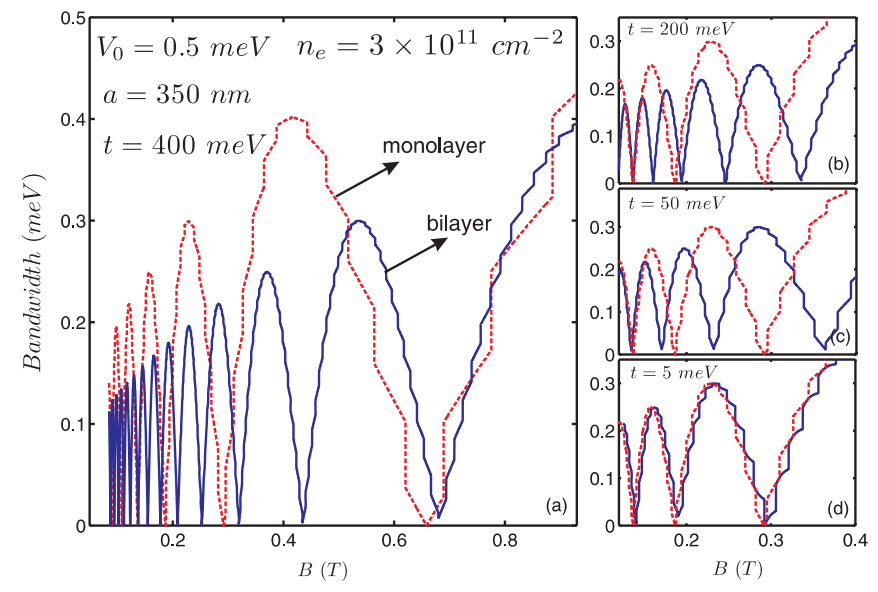

FIG. 2. (Color online) (a) The LL bandwidth at the Fermi energy as a function of the external magnetic field $B$ for the parameters shown. The dashed red and solid blue curves are for monolayer and bilayer graphene, respectively. (b)-(d) show the bandwidth for different values of the interlayer coupling $t$ as specified. For very small $t$, corresponding to nearly uncoupled layers, monolayer and bilayer results nearly coincide.

further reflected in Figs. 2(b)-2(d), where the region $0 \leqslant B \leqslant$ $0.4 \mathrm{~T}$ is plotted again for three different values of $t$. As shown in Fig. 2(d), for very small $t$, corresponding to nearly uncoupled layers, monolayer and bilayer results nearly coincide.

The LL bandwidth is also influenced by the phase between the potentials $V_{1}(x)$ and $V_{2}(x)$ : We show this influence in Fig. 3, where we plot the bandwidth versus (weak) field $B$ for cases (i)-(iii) mentioned above and schematically depicted at the top of the figure. Comparing curves (i) and (iii) makes clear that a phase shift (by $\pi$ ) between $V_{1}(x)$ and $V_{2}(x)$ changes the oscillation period and leads to an extremely severe reduction of the bandwidth; this reflects the fact that the "average" potential

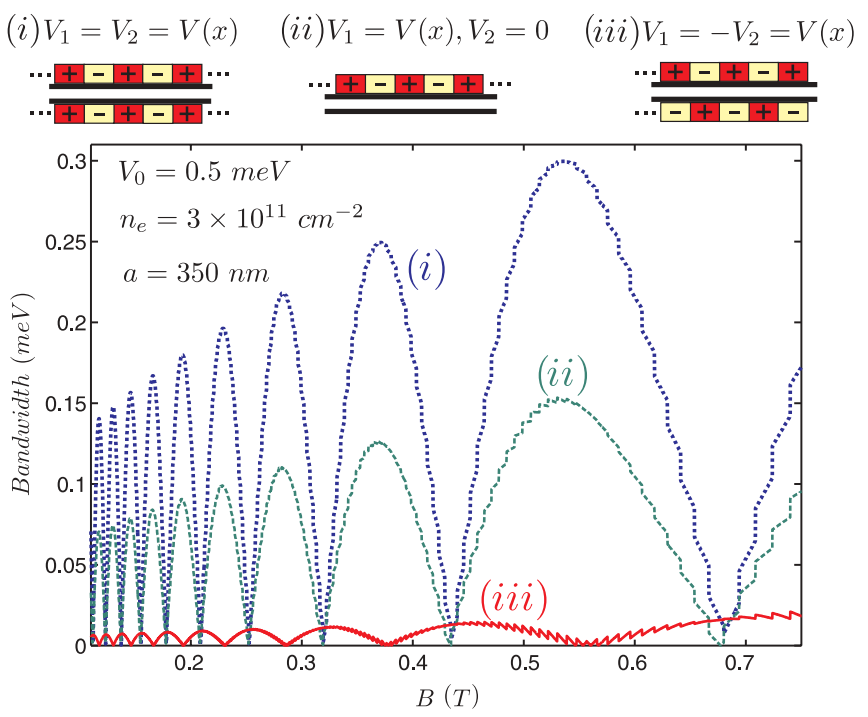

FIG. 3. (Color online) (a) The LL bandwidth at the Fermi energy, in bilayer graphene, as a function of the external magnetic field $B$ for the parameters shown in the inset. The curves (i), (ii), and (iii) correspond to the potentials $V_{1}(x)$ and $V_{2}(x)$, as specified in the text and schematically depicted at the top of the figure. applied to the bilayer is zero. This reduction is much less drastic if the phase shift is less than $\pi$ or if only one layer is modulated, as shown by curve (ii), in which case the oscillation period remains the same.

For $n \gg 1$, i.e., for weak magnetic fields, we have $\epsilon_{n} \gg 1$ and we can use the asymptotic expression of the Laguerre polynomials $L_{n}(u) \rightarrow\left(e^{u / 2} / \sqrt{\pi \sqrt{n u}}\right) \cos (2 \sqrt{n u}-\pi / 4)$. Then for case (i) the extrema of the bandwidth are given by

$$
B_{\mathrm{ex}}=\frac{2 \sqrt{E_{F}^{2}+E_{F} t}}{a(i+j / 4)}, \quad i=0,1,2, \ldots,
$$

with $j=1$ for the maximum and $j=3$ for the minimum. Note that this expression becomes the bandwidth extrema in single-layer graphene when $t=0$. For the parameters shown in Fig. 2, i.e., $n_{e}=3 \times 10^{-11} \mathrm{~cm}^{-2}$, $a=350 \mathrm{~nm}, V_{0}=0.5 \mathrm{meV}$, and $t=400 \mathrm{meV}$, we obtain the magnetic field values for zero bandwidth $B_{\min }(T)=$ $0.111,0.123,0.137,0.155,0.178,0.209, \ldots$ and for maximum bandwidth $\quad B_{\max }(T)=0.117,0.13,0.145,0.165,0.192$, $0.229, \ldots$. These values agree very well with those for the maxima and minima in the bandwidth shown in Fig. 2(a) that were obtained without using the asymptotic expression of the Laguerre polynomials. The behavior of the bandwidth for cases (ii) and (iii) is similar.

\section{A. Density of states}

The density of states (DOS) $D(E)$ is given by $D(E)=$ $(1 / 2 \pi) \sum_{n, s} \int d k_{y} \delta\left(E-E_{n, s, k_{y}}\right)$, where $E_{n, s, k_{y}}$ are the energy levels given by Eq. (9). To calculate it numerically we assume a Lorentzian broadening of zero shift and of width $\Gamma$. Since we focus on very weak magnetic fields, it is sufficient to take $\Gamma$ constant. We then have

$$
\begin{aligned}
D(E) / D_{0}= & \frac{1}{4 \pi^{2} E}\left(\frac{\hbar v_{F}}{l_{B}}\right)^{2} \sum_{n, s} \int_{0}^{2 \pi} \\
& \times \frac{\Gamma d \theta}{\Gamma^{2}+\left[E-E_{n, s}-V_{0} e^{-u / 2} G_{n, s} \cos \theta\right]^{2}},
\end{aligned}
$$

where $D_{0}=E / \pi\left(\hbar v_{F}\right)^{2}$ and $\theta=\left(2 \pi l_{B}^{2} / a\right) k_{y}$. Formally the same expression holds for single-layer graphene with appropriate changes to $E_{n, s}$ and $G_{n, s}$. For our numerical calculation we took $\Gamma=0.04 \mathrm{meV}$.

In Figs. 4(a) and 4(b) we plot the DOS for single-layer and case (i) bilayer graphene for fixed $B=0.1 \mathrm{~T}$; the other parameters are shown in the inset of Fig. 4(a). In both panels one sees the expected van Hove singularities (i.e., the double peak structure). In contrast with the modulated 2DEG, the peaks are not equidistant due to the difference in the energy spectra. This is more pronounced in Fig. 4(a) due to the absence of the interlayer coupling and the rather large value of $t$ [cf. Eq. (3)]. In Figs. 4(c) and 4(d) we plot the DOS versus the field $B$ for fixed energy $E=20 \mathrm{meV}$. In both cases the peaks are due to the van Hove singularities that are present at the edges of the modulation-broadened LLs. As can be seen, the peaks in the DOS of bilayer graphene occur at higher values of $B$. This is also reflected in the Hall and collisional conductivities of the next section. 

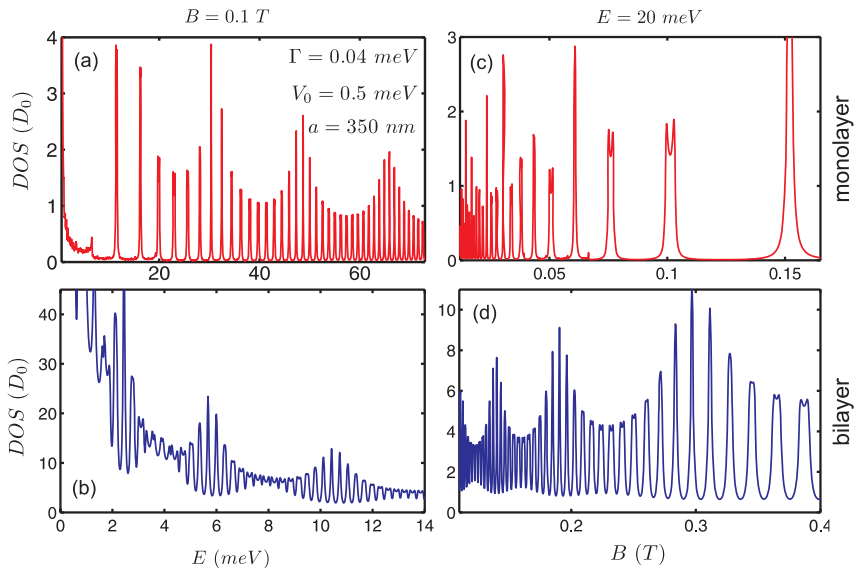

FIG. 4. (Color online) Density of states (in units of the zeromagnetic-field value $D_{0}$ ) of single-layer (upper panels) and bilayer [lower panels, case (i)] graphene. (a) and (b) show the DOS as a function of energy for $B=0.1 \mathrm{~T}$, whereas (c) and (d) show it as function of the magnetic field $B$ for $E=20 \mathrm{meV}$. The other parameters are shown in (a).

\section{CONDUCTIVITIES}

\section{A. Diffusive conductivity}

In the formalism followed in Ref. 2 the dc diffusive or band conductivity is given by

$$
\sigma_{\mu \nu}^{\mathrm{dif}}=\frac{4 \beta e^{2}}{L_{x} L_{y}} \sum_{\zeta} f_{\zeta}\left(1-f_{\zeta}\right) \tau_{\zeta} v_{\mu \zeta} v_{\nu \zeta} .
$$

Here $\mu, v=x, y, \beta=1 / k_{B} T,|\zeta\rangle \equiv\left|n, s, k_{y}\right\rangle, f_{\zeta} \equiv f\left(E_{\zeta}\right)$ is the Fermi-Dirac distribution, $E_{\zeta}$ the eigenvalue, $\tau_{\zeta}$ the relaxation time, and $v_{\mu \zeta}$ the diagonal matrix element of the velocity operator. The designation diagonal or off-diagonal refers to the eigenstates (2) and not to $\mu=v$ or $\mu \neq v$. The factor 4 in Eq. (13) accounts for valley and spin degeneracy. Note that Eq. (13) is valid only for elastic or quasielastic scattering. Further,

$$
\begin{aligned}
v_{y, \zeta} & \equiv v_{y, n, s, k_{y}}=\left(\partial E_{n, s, k_{y}} / \hbar \partial k_{y}\right) \\
& =-\left(2 V_{0} / \hbar K\right) u e^{-u / 2} \sin \left(K x_{0}\right) G_{n, s} .
\end{aligned}
$$

Since $\left\langle\psi\left|v_{x}\right| \psi\right\rangle=v_{x}=0$, the diffusive components $\sigma_{x x}^{\text {dif }}$ and $\sigma_{x y}^{\text {dif }}$ vanish identically. Due to the modulation, though, which broadens the LLs into bands, the component $\sigma_{y y}^{\text {dif }}$ does not vanish. Substituting $v_{y, \zeta}$ in Eq. (13) and integrating over $k_{y}\left(0 \leqslant k_{y} \leqslant L_{x} / \ell_{B}^{2}\right)$ the result for it is

$$
\sigma_{y y}^{\mathrm{dif}}=\frac{4 e^{2}}{h} \frac{V_{0}^{2} \tau}{\hbar} u e^{-u} \sum_{n=1, s} G_{n, s}^{2}[-\partial f(E) / \partial E]_{E=E_{n, s}},
$$

where $\tau=\mu E_{F} / e v_{F}^{2}$ with $\mu \approx 7 \times 10^{3} \mathrm{~cm}^{2} / \mathrm{Vs}$ a typical value for the bilayer graphene mobility. ${ }^{15}$

For case (i) Eq. (15), with $\sigma_{y y}^{\mathrm{dif}} \equiv \sigma_{y y}$, is plotted in Fig. 5 for (a) monolayer and (b) bilayer graphene, at temperatures $T=2$ and $6 \mathrm{~K}$. The parameters are shown in Fig. 5(b) and are the same with those in Fig. 5(a). The insets show $\sigma_{y y}$ for a higher electron density $\left(n_{e}=7 \times 10^{12} / \mathrm{cm}^{2}\right)$ for which both groups of LLs are occupied (cf. Fig. 1). At low $B$ we see the Weiss or commensurability oscillations, whereas at higher $B$

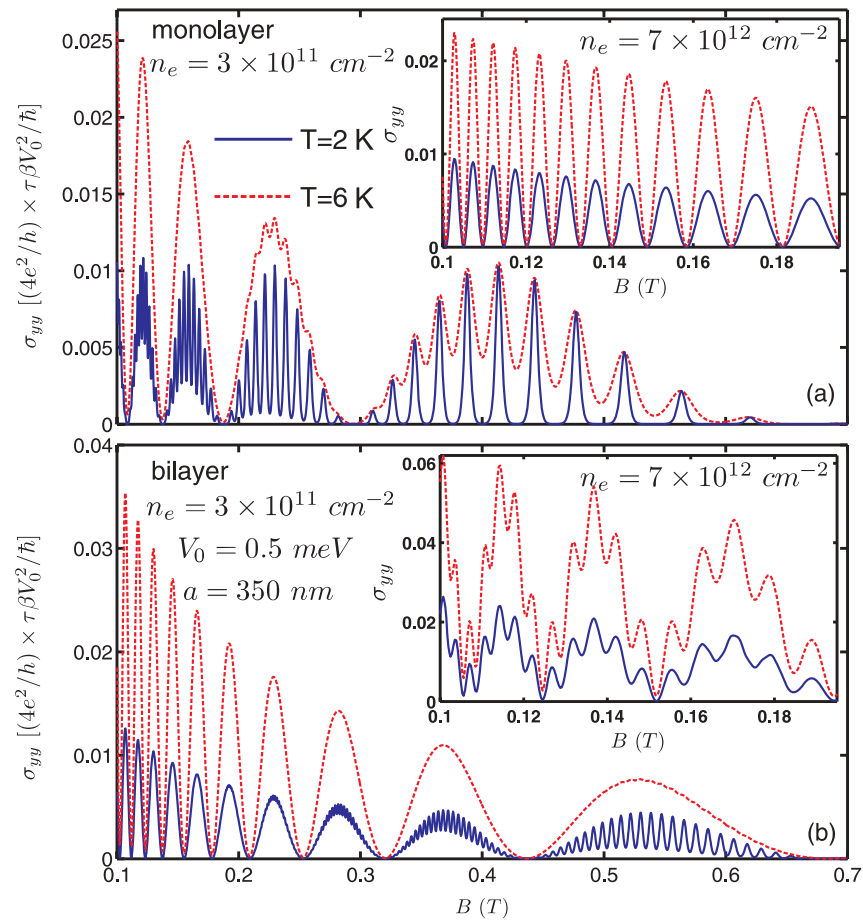

FIG. 5. (Color online) Diffusive conductivity $\sigma_{y y}$, as a function of the external magnetic field $B$, for (a) monolayer and (b) bilayer graphene [case (i)] at temperatures $T=2$ and $6 \mathrm{~K}$. The insets show $\sigma_{y y}$ for a higher electron density for which both groups of LLs are occupied (cf. Fig. 1).

the usual Shubnikov-de Haas oscillations start to appear, the former enveloping the latter. Again the amplitude in monolayer graphene is mostly larger than that in bilayer graphene.

The behavior of $\sigma_{y y}^{\text {dif }}$ for different potentials $V_{1}(x)$ and $V_{2}(x)$ applied to the two layers of bilayer graphene is similar to that in Fig. 5(b). We show that in Fig. 6, where we contrast the three cases depicted at the top of Fig. 3. As can be seen, the reduction of $\sigma_{y y}^{\text {dif }}$ follows that of the bandwidth shown in Fig. 3.

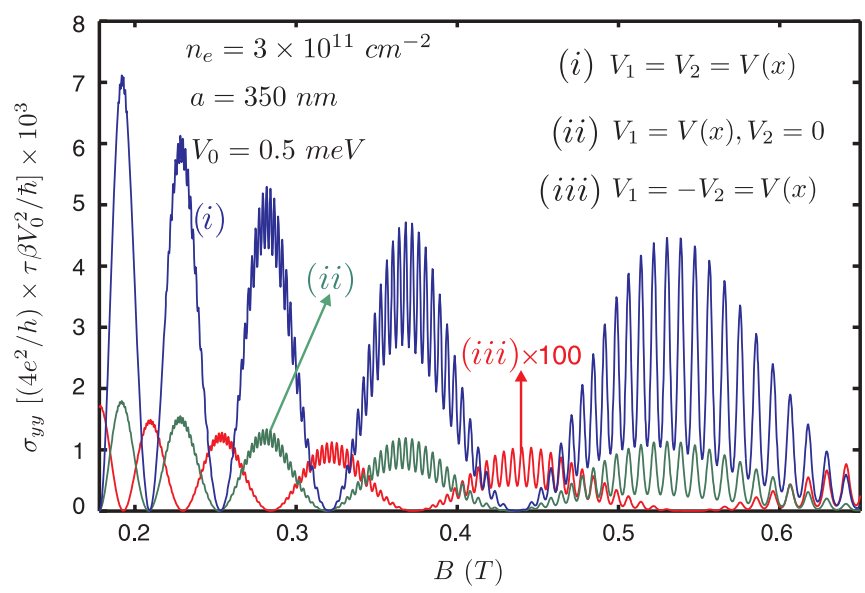

FIG. 6. (Color online) As in Fig. 5(b) for different potentials $V_{1}(x)$ and $V_{2}(x)$ applied to the two layers of bilayer graphene and specified after Eq. (5) and in the figure. The temperature is $T=2 \mathrm{~K}$. 


\section{B. Hall conductivity}

Within linear response theory the Hall conductivity is given by ${ }^{2}$

$$
\sigma_{y x}^{n d}=\frac{4 i \hbar e^{2}}{L_{x} L_{y}} \sum_{\zeta \neq \zeta^{\prime}} f_{\zeta}\left(1-f_{\zeta}^{\prime}\right)\left[1-e^{\beta\left(E_{\zeta}-E_{\zeta^{\prime}}\right)}\right] \frac{\left\langle\zeta\left|v_{x}\right| \zeta^{\prime}\right\rangle\left\langle\zeta^{\prime}\left|v_{y}\right| \zeta\right\rangle}{\left(E_{\zeta}-E_{\zeta^{\prime}}\right)^{2}},
$$

where $\left\langle\zeta\left|v_{x}\right| \zeta^{\prime}\right\rangle$ and $\left\langle\zeta^{\prime}\left|v_{y}\right| \zeta\right\rangle$ are the off-diagonal matrix elements of the velocity operator. They are evaluated with the help of the corresponding operators $v_{x}=\partial H_{0} / \partial p_{x}$ and $v_{y}=\partial H_{0} / \partial p_{y}$, given explicitly by

$$
v_{x}=v_{F}\left(\begin{array}{cc}
\sigma_{x} & 0 \\
0 & \sigma_{x}
\end{array}\right), \quad v_{y}=v_{F}\left(\begin{array}{cc}
-\sigma_{y} & 0 \\
0 & \sigma_{y}
\end{array}\right),
$$

with $\sigma_{\mu}$ being the Pauli matrices. The results are

$$
\begin{aligned}
\left\langle\zeta\left|v_{x}\right| \zeta^{\prime}\right\rangle & \equiv\left\langle n, s, k_{y}\left|v_{x}\right| n^{\prime}, s^{\prime}, k_{y}\right\rangle \\
& =-i v_{F} l_{B} 2^{n} n ! \sqrt{\pi} \frac{N_{n, s} N_{n^{\prime}, s^{\prime}}}{\epsilon_{n, s} \epsilon_{n^{\prime}, s^{\prime}}}\left\{\left[2(n+1) \epsilon_{n^{\prime}, s^{\prime}} k_{n, s} k_{n^{\prime}, s^{\prime}}+2 n^{\prime} \epsilon_{n, s}\right] \delta_{n, n^{\prime}-1}-\left[\epsilon_{n, s} k_{n, s} k_{n^{\prime}, s^{\prime}}+\epsilon_{n^{\prime}, s^{\prime}}\right] \delta_{n, n^{\prime}+1}\right\}, \\
\left\langle\zeta^{\prime}\left|v_{y}\right| \zeta\right\rangle & \equiv\left\langle n^{\prime}, s^{\prime}, k_{y}\left|v_{y}\right| n, s, k_{y}\right\rangle \\
& =v_{F} l_{B} 2^{n^{\prime}} n^{\prime} ! \sqrt{\pi} \frac{N_{n, s} N_{n^{\prime}, s^{\prime}}}{\epsilon_{n, s} \epsilon_{n^{\prime}, s^{\prime}}}\left\{\left[\epsilon_{n^{\prime}, s^{\prime}} k_{n, s} k_{n^{\prime}, s^{\prime}}+\epsilon_{n, s}\right] \delta_{n, n^{\prime}-1}+\left[2 n \epsilon_{n^{\prime}, s^{\prime}}+2\left(n^{\prime}+1\right) \epsilon_{n, s} k_{n, s} k_{n^{\prime}, s^{\prime}}\right] \delta_{n, n^{\prime}+1}\right\},
\end{aligned}
$$

where $s=\left\{s_{1}, s_{2}\right\}$ and $s^{\prime}=\left\{s_{1}^{\prime}, s_{2}^{\prime}\right\}$. Using the identity $f_{\zeta}\left(1-f_{\zeta^{\prime}}\right)\left[1-e^{\beta\left(E_{\zeta}-E_{\zeta^{\prime}}\right)}\right]=f_{\zeta}-f_{\zeta^{\prime}}$, inserting Eqs. (18) and (19) in Eq. (16), and setting $\theta=\left(2 \pi l_{B}^{2} / a\right) k_{y}$, we obtain

$$
\begin{aligned}
\sigma_{y x}= & \frac{4 e^{2}}{h} \frac{\hbar^{2} v_{F}^{2}}{\pi l_{B}^{2}} \sum_{s, s^{\prime}} \sum_{n}^{\infty}\left\{\eta_{n, s, s^{\prime}} \int_{0}^{2 \pi} \frac{\left(f_{n, s}-f_{n+1, s^{\prime}}\right) d \theta}{\left[E_{n, s}-E_{n+1, s^{\prime}}+\left(\lambda_{n, s}-\lambda_{n+1, s^{\prime}}\right) \cos \theta\right]^{2}}\right. \\
& \left.-\xi_{n, s, s^{\prime}} \int_{0}^{2 \pi} \frac{\left(f_{n, s}-f_{n-1, s^{\prime}}\right) d \theta}{\left[E_{n, s}-E_{n-1, s^{\prime}}+\left(\lambda_{n, s}-\lambda_{n-1, s^{\prime}}\right) \cos \theta\right]^{2}}\right\},
\end{aligned}
$$

where $\lambda_{n, s}=V_{0} e^{-u / 2} G_{n, s}$ and

$$
\begin{aligned}
& \eta_{n, s, s^{\prime}}=(n+1)\left[\frac{d_{n, s} d_{n+1, s^{\prime}}}{\epsilon_{n, s} \epsilon_{n+1, s^{\prime}}}\left(k_{n} k_{n+1, s^{\prime}} \epsilon_{n+1, s^{\prime}}+\epsilon_{n, s}\right)\right]^{2}, \\
& \xi_{n, s, s^{\prime}}=n\left[\frac{d_{n, s} d_{n-1, s^{\prime}}}{\epsilon_{n, s} \epsilon_{n-1, s^{\prime}}}\left(k_{n} k_{n-1, s^{\prime}} \epsilon_{n, s}+\epsilon_{n-1, s^{\prime}}\right)\right]^{2} .
\end{aligned}
$$

Due to the $n-1$ subscripts, the second term in Eq. (20) is valid only for $n \geqslant 2$, whereas the first term is valid for $n \geqslant 1$. However, one can change $n-1$ to $n$ in the second term and combine it with the $n \geqslant 1$ term. Then the sum over $n$ starts at $n=1$ for both terms. If only the $n=0 \mathrm{LL}$ is occupied the Hall conductivity can be evaluated using the $n=0$ eigenstates given in Appendix B. The result is given by Eq. (B6). When the $n$th LL is occupied, $n \geqslant 1$, at $T=0$ this $n=0$ contribution vanishes identically since all Fermi factors are equal to 1 .

In Fig. 7 we contrast the Hall conductivities versus (weak) magnetic field $B$ in monolayer and case (i) bilayer graphene. The result for the former is taken from a straightforward extension of Eq. (14) of Ref. 16, valid for $V_{0}=0$, to the case where the modulation is present $\left(V_{0} \neq 0\right)$ [see also Eq. (B7)]. Surprisingly, one can see well-defined plateaus at (a) half-integer and (b) integer multiples of $4 e^{2} / h$ although the field $B$ is weak. Notice that for bilayer graphene the plateaus occur at higher $B$ than in monolayer graphene. This is due to the shift in the energy levels brought about by the interlayer coupling $t$ in bilayer graphene [cf. Eq. (3) and Sec. III]. An important feature is that at $T=6 \mathrm{~K}$ the plateaus have almost disappeared. This is because neither the $k$-dependent shift of the LLs nor their width have been taken into account. If one takes them into account, something that is beyond the scope of the present work where we focus on weak $B$ and weak modulation, the temperature range of well-defined plateaus can be increased by about one order of magnitude (cf. Ref. 16).

Another noteworthy feature of the Hall conductivities shown in Fig. 7, is their behavior at very low $B$ fields for which the plateaus are not resolved. Their apparent monotonic behavior with $B$ is in fact an oscillatory one as shown in Figs. 8(a) and $8(\mathrm{~b})$, where we plot the derivative $d \sigma_{y x} / d B$ for very weak fields. As inferred, in these ranges of $B$ the Hall conductivity $\sigma_{y x}$ oscillates with $B$ but its small-amplitude oscillations are not visible on the scale of Fig. 7, whereas they are in $d \sigma_{y x} / d B$ shown in Fig. 8. Notice that the Hall conductivity for the bilayer cases (ii) and (iii), with different applied voltages $V_{1}(x)$ and $V_{2}(x)$, leads to the same results as case (i). This is due to the subtraction of the bandwidths of adjacent LLs described by the term $\lambda_{n, s}-\lambda_{n \pm 1, s^{\prime}}$ in Eq. (20) and to the smallness of the energy correction, due to the modulation, compared to the Fermi level. 


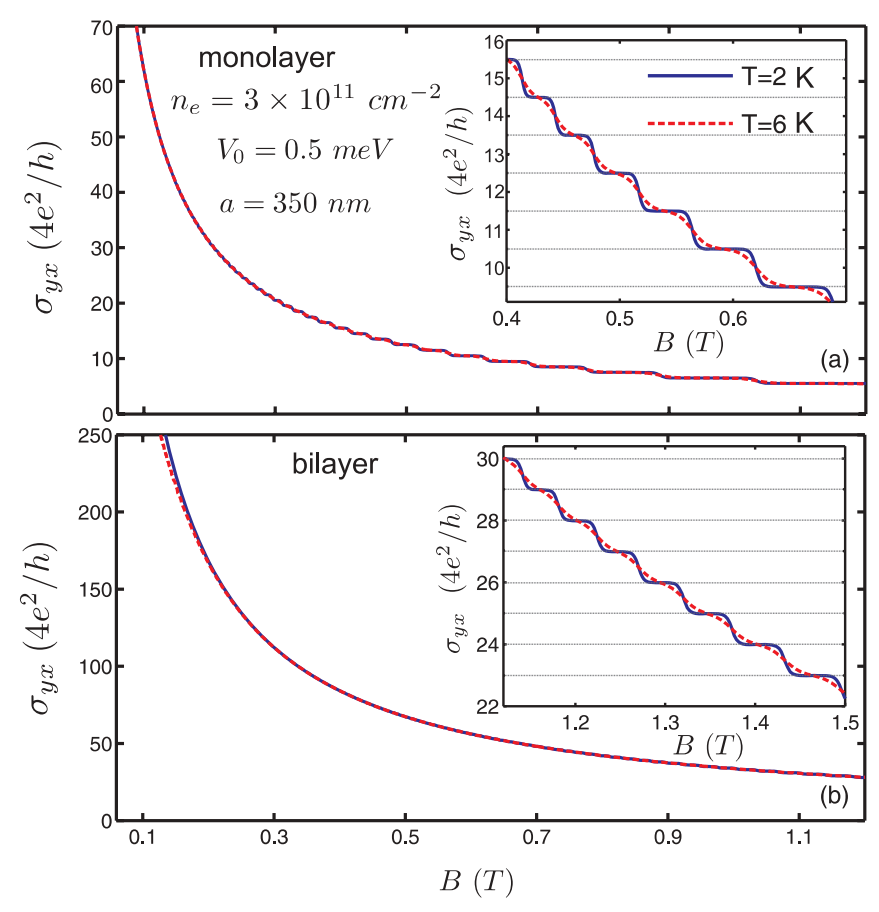

FIG. 7. (Color online) Hall conductivity $\sigma_{y x}$, in (a) single-layer and (b) bilayer graphene [case (i)], as a function of an external magnetic field $B$, at temperatures $T=2$ and $6 \mathrm{~K}$. The insets show $\sigma_{y x}$ in a narrow range of $B$. Notice the plateaus, for $T=2 \mathrm{~K}$, at half-integer and integer multiples of $4 e^{2} / h$ in (a) and (b), respectively, and their near disappearance at $T=6 \mathrm{~K}$.

\section{Collisional conductivity}

For the unmodulated 2DEG this is the only component that gives rise to the Shubnikov-de Haas oscillations since in this case $\sigma_{\mu \nu}^{\mathrm{dif}}$ vanishes identically. Taking spin and valley
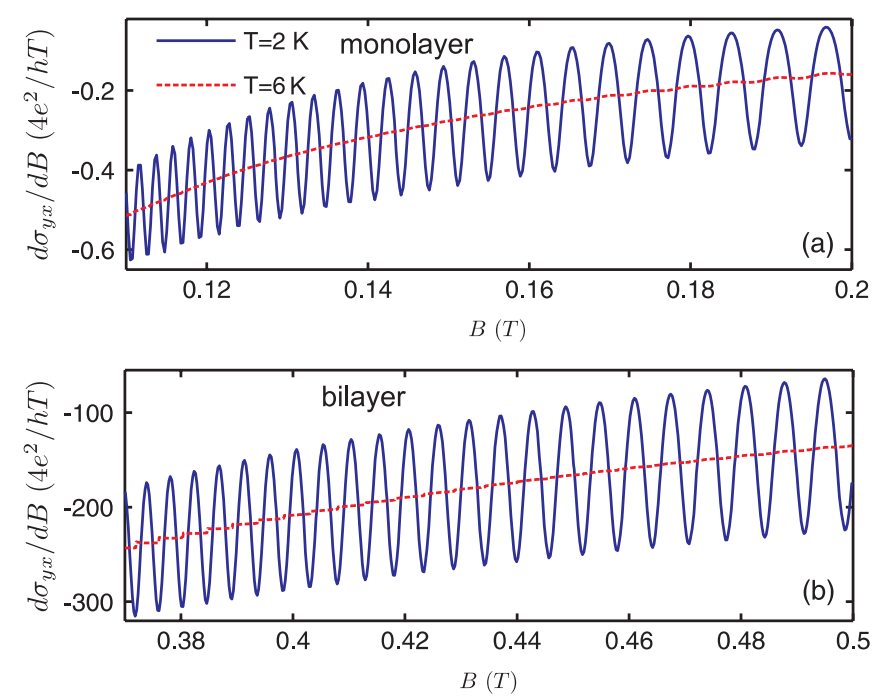

FIG. 8. (Color online) Derivative $d \sigma_{y x} / d B$ vs $B$ for very weak fields, for which the plateaus are not resolved, and two different temperatures. (a) is for single-layer and (b) for case (i) bilayer graphene. degeneracies into account it is given $(\mu=\nu)$ by

$$
\sigma_{\mu \mu}^{\mathrm{col}}=\frac{2 \beta e^{2}}{L_{x} L_{y}} \sum_{\zeta, \zeta^{\prime}} f\left(E_{\zeta}\right)\left[1-f\left(E_{\zeta^{\prime}}\right)\right] W_{\zeta, \zeta^{\prime}}\left(a_{\mu, \zeta}-a_{\mu, \zeta^{\prime}}\right)^{2},
$$

where $W_{\zeta, \zeta^{\prime}}$ is the transition rate between the states $\zeta$ and $\zeta^{\prime}$ and $a_{\mu, \zeta}=\left\langle\zeta\left|r_{\mu}\right| \zeta\right\rangle$ the expectation value of the position operator. In contrast with Eqs. (13) and (22) is valid for both elastic and inelastic scattering.

The evaluation of Eq. (22) is carried out along the lines of Ref. 2. Within first-order perturbation theory the perturbed eigenfunctions $\left|n, s, k_{y}\right\rangle$ are given by

$$
\left|n, s, k_{y}\right\rangle=\left|n, s, k_{y}\right\rangle^{0}+\sum_{n^{\prime} \neq n} \frac{V_{n, s, n^{\prime}, s^{\prime}}}{E_{n, s}-E_{n^{\prime}, s}}\left|n^{\prime}, s^{\prime}, k_{y}\right\rangle^{0},
$$

with $\left.\left|n, s, k_{y}\right|\right\rangle^{0}$ being the unperturbed ones given by Eq. (4).

For the evaluation of Eq. (22) using the states (23) we assume elastic scattering by random impurities of density $N_{I}$. We first notice that in Eq. (22) we have, for $\mu=y$, $a_{y, \zeta}-a_{y, \zeta^{\prime}}=0$. That is, $\sigma_{y y}^{\text {col }}$ vanishes and we have to evaluate only the component $\sigma_{x x}^{\text {col }}$. Because the details are rather involved, we present them in Appendix $C$. With $A_{0}=\left(2 e^{2} / h\right)\left(N_{I} U_{0}^{2} / \pi^{2} l_{B}^{2} \Gamma\right)$ the result for $\sigma_{x x}^{\text {col, } 0}$, without the correction to the wave function, is

$$
\sigma_{x x}^{\mathrm{col}, 0} \approx A_{0} \sum_{n, s}^{\infty} S_{n, s} I_{n, s},
$$

where the level width $\Gamma$ is taken constant due to the smallness of $B . S_{n}$ is given by Eq. (C8) and

$$
I_{n, s}=\int_{0}^{2 \pi}\left[-\partial f\left(E_{n, s, \theta}\right) / \partial E_{n, s, \theta}\right] d \theta .
$$

If we include the correction to the unperturbed wave function the total collisional conductivity takes the form

$$
\sigma_{x x}^{\mathrm{col}} \approx A_{0} \sum_{n, s}^{\infty} S_{n, s}\left[I_{n, s}+\Delta_{n, s} J_{n, s}\right]
$$

with $\Delta_{n}=2 V_{0}^{2} u e^{-u}\left[P_{n, s}^{2}+P_{n, s} P_{n+1, s}+P_{n+1, s}^{2}\right]$, the factor $P_{n}$ given by Eq. (C9), and

$$
J_{n, s}=\int_{0}^{2 \pi} \sin ^{2} \theta\left[-\partial f\left(E_{n, s, \theta}\right) / \partial E_{n, s, \theta}\right] d \theta .
$$

In Fig. 9 we plot $\sigma_{x x}^{\text {col }}$, for case (i) bilayer graphene, as a function of the external magnetic field $B$, at temperatures $T=2 \mathrm{~K}$ (blue solid curve) and $T=6 \mathrm{~K}$ (red dashed curve). As can be seen, this component oscillates with $B$ but the oscillation amplitude is much smaller than that of the diffusive contribution $\sigma_{y y}^{\text {dif }}$ shown in Fig. 5(b). Qualitatively this is also the case in a 2DEG and the oscillations reflect those of the DOS (cf. Ref. 2). To make this point clearer, the inset shows the collisional $\sigma_{x x}^{\text {col }}$ (blue solid curve) and $\sigma_{y y}^{\text {dif }}$ diffusive conductivities (dashed curve) on top of each other in a narrow range of low $B$. As shown, the two components oscillate out of phase. Similar results occur in single-layer graphene (see Ref. 10). 


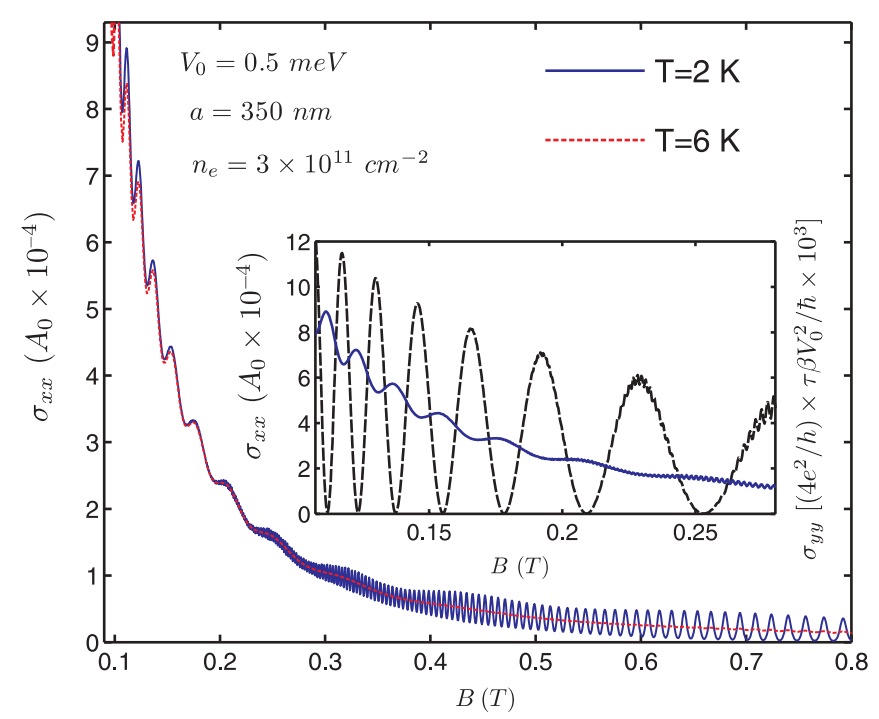

FIG. 9. (Color online) Collisional conductivity $\sigma_{x x}$, in bilayer graphene, as a function of an external magnetic field $B$, at temperatures $T=2 \mathrm{~K}$ and $T=6 \mathrm{~K}$. The inset shows the collisional $\sigma_{x x}^{\text {col }}$ (blue solid curve) and $\sigma_{y y}^{\text {dif }}$ diffusive conductivities (dashed curve) on top of each other in a narrow range of low $B$. The two components oscillate out of phase.

\section{CONCLUDING REMARKS}

We evaluated the conductivity tensor in weakly and periodically modulated bilayer graphene in the presence of a perpendicular magnetic field $B$. As in the case of a 2DEG, the modulation broadens the Landau levels into bands and for weak magnetic fields leads to the Weiss oscillations in their bandwidth and the transport coefficients at very low $B$ and to the Shubnikov-de Haas oscillations at larger $B$. We also considered the case in which the periodic, sinusoidal potentials applied to the bilayer differ in phase by $\pi$ and the one in which such a potential is applied to only one layer. As expected, in the former case we have a severe reduction in the oscillation amplitude of the Weiss oscillation and a change in their period, whereas in the latter we have only a reduction in the oscillation amplitude.

We also contrasted some of the results, whenever deemed appropriate, with those of the literature pertinent to singlelayer graphene. As we specified in Sec. III, the corresponding flat-band condition, oscillation frequency, and oscillation amplitude differ substantially due to the interlayer coupling. In general, the oscillation amplitude and frequency are higher in single-layer graphene. As expected though and demonstrated in Fig. 2, the two results agree only when this coupling is extremely weak and the two layers are nearly independent.

Further, we showed that the Hall conductivity exhibits the well-known steps at half-integer and integer multiples of $e^{2} / \hbar$ in single-layer and bilayer graphene, respectively, though the magnetic fields involved are very weak. This is due to the larger energy scales, relative to those in a 2DEG, involved in graphene. For the same densities though we showed that the plateaus occur at quite different values of $B$ again due to the changes in the energy spectra and density of states brought about by the interlayer coupling. In addition, we showed that the collisional conductivity oscillates with the magnetic field
$B$ even for very weak values of $B$ close to $0.1 \mathrm{~T}$ in single-layer graphene and to $0.25 \mathrm{~T}$ in bilayer graphene (cf. Fig. 9).

Our study was limited to potential (or electric) modulations and did not include any periodic modulations of the magnetic field. However, on account of similar studies in a $2 \mathrm{DEG}^{3,4}$ and in single-layer graphene, 7,10 the results for magnetic modulations should be qualitatively similar. The same should hold especially when both types of modulations are present. ${ }^{3}$ Given that, our results are pertinent to weak and periodic corrugations of graphene ${ }^{8}$ when the potential modulation dominates the stress-induced magnetic modulation.

Note added. We just became aware that the Weiss oscillations and van Hove singularities have been experimentally observed in graphene-based systems subjected to twodimensional periodic modulations. ${ }^{17}$

\section{ACKNOWLEDGMENTS}

This work was supported by the Flemish Science Foundation (FWO-Vl), the European Science Foundation (ESF) under the EUROCORES program EuroGRAPHENE (project CONGRAN), and the Canadian NSERC Grant No. OGP0121756.

\section{APPENDIX A: EIGENVALUES AND EIGENSTATES FOR ZERO BIAS}

Below we obtain the eigenfunctions and eigenvalues of the Hamiltonian (1) following Ref. 13 and neglecting the Zeeman term due to its smallness. Using Eqs. (1) and (2) $H \Psi=E \Psi$ leads to

$$
\begin{gathered}
(d / d x-x) \varphi_{b}=\epsilon \varphi_{a}-t \varphi_{c}, \\
-(d / d x+x) \varphi_{a}=\epsilon \varphi_{b}, \\
(d / d x+x) \varphi_{d}=\epsilon \varphi_{c}-t \varphi_{a}, \\
-(d / d x-x) \varphi_{c}=\epsilon \varphi_{d},
\end{gathered}
$$

where we used the dimensionless units $x \rightarrow l_{B} k_{y}+x / l_{B}$, $\epsilon=\left(l_{B} / \hbar v_{F}\right) E, t \rightarrow\left(l_{B} / \hbar v_{F}\right) t$. Equations (A1)-(A4) can be reduced to the coupled differential equations

$$
\begin{aligned}
& {\left[d^{2} / d x^{2}-x^{2}+1+\epsilon^{2}\right] \varphi_{a}=t \epsilon \varphi_{c},} \\
& {\left[d^{2} / d x^{2}-x^{2}-1+\epsilon^{2}\right] \varphi_{c}=t \epsilon \varphi_{a} .}
\end{aligned}
$$

Decoupling Eqs. (A5) results in

$$
\left(d^{2} / d x^{2}-x^{2}+\epsilon^{2}\right)^{2} \varphi_{a}=\left[1+t^{2} \epsilon^{2}\right] \varphi_{a} .
$$

We note that the solution of the following equation can also satisfy Eq. (A6),

$$
\left(d^{2} / d x^{2}-x^{2}\right) \varphi_{a}=\gamma_{ \pm} \varphi_{a}
$$

where $\gamma_{ \pm}=-\epsilon^{2} \pm\left[1+t^{2} \epsilon^{2}\right]^{1 / 2}$. To solve Eq. (A7) we set $\varphi_{a}(x)=f(x) e^{-x^{2} / 2}$; this eliminates the $x^{2}$ term and gives

$$
\frac{d^{2} f}{d x^{2}}-2 x \frac{d f}{d x}-\left(\gamma_{ \pm}+1\right) f=0 .
$$


This is the equation satisfied by the Hermite polynomials $H_{n}(x)$ for $1+\gamma_{ \pm}=-2 n$. The resulting eigenvalues are

$$
\epsilon=s_{1}\left\{t^{2} / 2+2 n+1+s_{2} \sqrt{t^{4} / 4+(2 n+1) t^{2}+1}\right\}^{1 / 2}, \quad n=0,1,2, \ldots,
$$

where $s_{1}= \pm 1$ and $s_{2}= \pm 1$ give the different energy bands. The corresponding spinor $\varphi_{a}$ is

$$
\varphi_{a}=N e^{-x^{2} / 2} H_{n}(x),
$$

where $N$ is a normalization factor. Substituting $\varphi_{a}$ in Eq. (A2) and using the relation $d H_{n}(x) / d x=2 n H_{n-1}(x)$ gives

$$
\varphi_{b}=-(2 n / \epsilon) N e^{-x^{2} / 2} H_{n-1}(x) .
$$

The resulting $\varphi_{c}$ and $\varphi_{d}$ are

$$
\varphi_{c}=k_{n} N e^{-x^{2} / 2} H_{n}(x), \quad \varphi_{d}=\left(k_{n} / \epsilon\right) N e^{-x^{2} / 2} H_{n+1}(x),
$$

with $k_{n}=\left(\epsilon^{2}-2 n\right) / t \epsilon$. The normalization of the spinors, upon using

$$
\int_{-\infty}^{\infty}\left[\varphi_{a}^{*} \varphi_{a}+\varphi_{b}^{*} \varphi_{b}+\varphi_{c}^{*} \varphi_{c}+\varphi_{d}^{*} \varphi_{d}\right] d x=1
$$

gives

$$
N \equiv N_{n}=d_{n} /\left(2^{n} n ! \sqrt{\pi} l_{B}\right)^{1 / 2},
$$

with $d_{n}=\left[k_{n}^{2}\left[1+2(n+1) / \epsilon^{2}\right]+1+2 n / \epsilon^{2}\right]^{-1 / 2}$.

\section{APPENDIX B: ZERO-ENERGY SOLUTION}

Equations (A10)-(A12) and (A14) are valid for $n \geqslant 1$. For $n=0$ and $\epsilon=0$ Eqs. (A1)-(A4) take a simpler form. A solution for the normalized eigenfunctions is

$$
\begin{aligned}
& \psi(x)_{0^{+-}}=\frac{1}{\left[L_{y} l_{B} \sqrt{\pi}\left(1+t^{2} / 2\right)\right]^{1 / 2}}\left(\begin{array}{c}
e^{-x^{2} / 2} H_{0}(x) \\
0 \\
0 \\
-t e^{-x^{2} / 2} H_{1}(x) / 2
\end{array}\right) e^{i k_{y} y}, \\
& \psi(x)_{0^{--}}=\frac{1}{\sqrt{L_{y} l_{B} \sqrt{\pi}}}\left(\begin{array}{c}
0 \\
0 \\
0 \\
e^{-x^{2} / 2} H_{0}(x)
\end{array}\right) e^{i k_{y} y} .
\end{aligned}
$$

This solution agrees with that of Ref. 18. Notice that $\psi(x)=\left[\varphi_{a}, i \varphi_{b}, \varphi_{c}, i \varphi_{d}\right]^{T}$. Using Eqs. (B1) and (B2) we obtain the velocity matrix elements

$$
\left\langle 0_{+}\left|v_{x}\right| n^{\prime}\right\rangle=-i v_{F} \frac{d_{n^{\prime}, s^{\prime}}\left(2^{n^{\prime}} n^{\prime} !\right)^{1 / 2}}{\left(1+t^{2} / 2\right)^{1 / 2}}\left[\frac{1}{\epsilon_{n^{\prime}, s^{\prime}}}-\frac{t k_{n^{\prime}, s^{\prime}}}{2}\right] \delta_{1, n^{\prime}},
$$

and $\left\langle n^{\prime}\left|v_{y}\right| 0_{+}\right\rangle=i\left\langle 0_{+}\left|v_{x}\right| n^{\prime}\right\rangle$. For $\left|0_{-}\right\rangle$we have

$$
\left\langle 0_{-}\left|v_{x}\right| n^{\prime}\right\rangle=-i v_{F} d_{n^{\prime}, s^{\prime}}\left(2^{n^{\prime}} n^{\prime} !\right)^{1 / 2} k_{n^{\prime}, s^{\prime}} \delta_{0, n^{\prime}},
$$

with $\left\langle n^{\prime}\left|v_{y}\right| 0_{-}\right\rangle=i\left\langle 0_{-}\left|v_{x}\right| n^{\prime}\right\rangle$. In addition, we have $\epsilon_{0, s_{1}^{\prime},-}=0, \epsilon_{0, s_{1}^{\prime},+}=s_{1}^{\prime} \sqrt{t^{2}+2}$, and

$$
\lim _{n^{\prime} \rightarrow 0} d_{n^{\prime}, s_{1}^{\prime},-}^{2} k_{n^{\prime}, s_{1}^{\prime},-}^{2}=0, \quad \lim _{n^{\prime} \rightarrow 0} d_{n^{\prime}, s_{1}^{\prime},+}^{2} k_{n^{\prime}, s_{1}^{\prime},+}^{2}=1 / 2 .
$$

Then the Hall conductivity is given by

$$
\sigma_{y x}=\frac{4 \hbar e^{2} v_{F}^{2}}{L_{x} L_{y}} \sum_{k_{y}}\left[\frac{4 d_{1,+,-}^{2}}{2+t^{2}}\left(\frac{1}{\epsilon_{1,+,-}}-\frac{t k_{1,+,-}}{2}\right)^{2} \frac{f_{0,+,-}-f_{1,+,-}}{\left(\epsilon_{0,+,-, k_{y}}-\epsilon_{1,+,-, k_{y}}\right)^{2}}+\frac{1}{2} \frac{f_{0,-,-}-f_{0,-,+}}{\left(\epsilon_{0,-,-, k_{y}}-\epsilon_{0,-,+, k_{y}}\right)^{2}}\right] .
$$

For $t \rightarrow 0$ we have $d_{n^{\prime}, s_{1}^{\prime}, s_{2}^{\prime}}=1 / \sqrt{2}, \epsilon_{0, \pm,-}=0, \epsilon_{1,+,-}=\epsilon_{0,+,+}=\sqrt{2}$, and $\epsilon_{0,-,+}=\epsilon_{1,-,-}=\sqrt{2}$. Then Eq. (B6) leads to

$$
\sigma_{y x}=\frac{\hbar e^{2} v_{F}^{2}}{L_{x} L_{y}} \sum_{k_{y}}\left[f_{0,+,-}-f_{1,+,-}+f_{0,-,-}-f_{1,-,-}\right] .
$$

In the absence of modulation $\left(\sum_{k_{y}} \rightarrow L_{x} L_{y} / \ell_{B}^{2}\right)$ Eq. (B7) gives the single-layer result, Eq. (14) of Ref. 16 for $n=0$. 


\section{APPENDIX C: COLLISIONAL CONDUCTIVITY}

Below we briefly present the evaluation of the collisional conductivity using Eqs. (23) and (24). For the evaluation of the transition rate $W_{\zeta \zeta^{\prime}}$, appearing in Eq. (22), we need the integral

$$
V_{n, s, n^{\prime}, s^{\prime}}={ }^{0}\left\langle n, s, k_{y}\left|V_{0} \cos (K x)\right| n^{\prime}, s^{\prime}, k_{y}\right\rangle^{0}=V_{0}(-1)^{m} F_{n, s, n^{\prime}, s^{\prime}} \begin{cases}\sqrt{2} \sin \left(K x_{0}\right), & n^{\prime}-n=2 m+1, \\ \cos \left(K x_{0}\right), & n^{\prime}-n=2 m,\end{cases}
$$

where

$$
F_{n, n^{\prime}}=d_{n, s} d_{n^{\prime}, s^{\prime}} \sqrt{\frac{2^{n} n !}{2^{n^{\prime}} n^{\prime} !}} u^{n^{\prime}-n} e^{-u / 2}\left[\left(1+k_{n, s} k_{n^{\prime}, s^{\prime}}\right) L_{n}^{n^{\prime}-n}(u)+\frac{2 n^{\prime}}{\epsilon_{n, s} \epsilon_{n^{\prime}, s^{\prime}}} L_{n-1}^{n^{\prime}-n}(u)+\frac{2(n+1) k_{n, s} k_{n^{\prime}, s^{\prime}}}{\epsilon_{n, s} \epsilon_{n^{\prime}, s^{\prime}}} L_{n+1}^{n^{\prime}-n}(u)\right] .
$$

As for the integral ${ }^{0}\left\langle n, k_{y}\left|e^{i \boldsymbol{q} \cdot \boldsymbol{r}}\right| n^{\prime}, k_{y}^{\prime}\right\rangle^{0}$, involving products of Hermite polynomials $H_{n}(x) H_{m}(x)$, it can be evaluated using the tabulated formula $\int_{-\infty}^{\infty} e^{-(x-y)^{2}} H_{n}(x) H_{m}(x) d x=2^{n} \sqrt{\pi} m ! y^{n-m} L_{m}^{n-m}\left(-2 y^{2}\right), m \leqslant n$. For $n^{\prime} \leqslant n$ we have

$$
\begin{aligned}
\left\langle n, s, k_{y}\left|e^{i \boldsymbol{q} \cdot \boldsymbol{r}}\right| n^{\prime}, s^{\prime}, k_{y}^{\prime}\right\rangle^{0}= & \delta_{k_{y}^{\prime}, q_{y}-k_{y}} e^{-i \Theta-\gamma / 2} v^{n-n^{\prime}} \sqrt{\frac{2^{n} n^{\prime} !}{2^{n^{\prime}} n !}} d_{n, s} d_{n^{\prime}, s^{\prime}} \\
& \times\left[\left(1+k_{n, s} k_{n^{\prime}, s^{\prime}}\right) L_{n^{\prime}}^{n-n^{\prime}}(\gamma)+\frac{2 n}{\epsilon_{n, s} \epsilon_{n^{\prime}, s^{\prime}}} L_{n^{\prime}-1}^{n-n^{\prime}}(\gamma)+\frac{2\left(n^{\prime}+1\right) k_{n} k_{n^{\prime}}}{\epsilon_{n, s} \epsilon_{n^{\prime}, s^{\prime}}} L_{n^{\prime}+1}^{n-n^{\prime}}(\gamma)\right],
\end{aligned}
$$

and for $n \leqslant n^{\prime}$

$$
\begin{aligned}
{ }^{0}\left\langle n, s, k_{y}\left|e^{i \boldsymbol{q} \cdot \boldsymbol{r}}\right| n^{\prime}, s^{\prime}, k_{y}^{\prime}\right\rangle^{0}= & \delta_{k_{y}^{\prime}, q_{y}-k_{y}} e^{-i \Theta-\gamma / 2} v^{n^{\prime}-n} \sqrt{\frac{2^{n^{\prime}} n !}{2^{n} n^{\prime} !}} d_{n, s} d_{n^{\prime}, s^{\prime}} \\
& \times\left[\left(1+k_{n, s} k_{n^{\prime}, s^{\prime}}\right) L_{n}^{n^{\prime}-n}(\gamma)+\frac{2 n^{\prime}}{\epsilon_{n, s} \epsilon_{n^{\prime}, s^{\prime}}} L_{n-1}^{n^{\prime}-n}(\gamma)+\frac{2(n+1) k_{n, s} k_{n^{\prime}, s^{\prime}}}{\epsilon_{n, s} \epsilon_{n^{\prime}, s^{\prime}}} L_{n+1}^{n^{\prime}-n}(\gamma)\right],
\end{aligned}
$$

where $\gamma=\left(q_{x}^{2}+q_{y}^{2}\right) l_{B}^{2} / 2, \Theta=x_{0}+q_{x} q_{y} l_{B}^{2} / 2$, and $v=\left(q_{y}+i q_{x}\right) l_{B} / 2$.

The screened impurity potential is given by $U(\boldsymbol{r}-\boldsymbol{R})=e^{2} e^{-k_{s}|\boldsymbol{r}-\boldsymbol{R}|} / \varepsilon|\boldsymbol{r}-\boldsymbol{R}|$ and its Fourier transform is $U_{q}=\left(2 \pi e^{2} / \varepsilon \sqrt{q^{2}+k_{s}^{2}}\right)$. Then the transition rate for randomly distributed impurities of density $N_{I}$ takes the form

$$
W_{\zeta \zeta^{\prime}}=\sum_{q}\left|U_{q}\right|^{2}\left|\left\langle n, k_{y}\left|e^{i \boldsymbol{q} \cdot \boldsymbol{r}}\right| n^{\prime}, k_{y}^{\prime}\right\rangle^{0}\right|^{2} \delta\left(\epsilon_{n, k_{y}}-\epsilon_{n^{\prime}, k_{y}^{\prime}}\right)=\left.\frac{2 \pi N_{I}}{L_{x} L_{y} \hbar} \sum_{q}\left|U_{q}\right|^{2}|| J_{n n^{\prime} s s^{\prime}}\right|^{2} \delta\left(\epsilon_{n, s, k_{y}}-\epsilon_{n^{\prime}, s^{\prime}, k_{y}^{\prime}}\right) \delta_{q_{y}, k_{y}^{\prime}-k_{y}},
$$

with

$$
\left|J_{n n^{\prime} s s^{\prime}}\right|^{2}=\frac{n^{\prime} !}{n !} d_{n, s} d_{n^{\prime}, s^{\prime}} e^{-\gamma} \gamma^{n-n^{\prime}}\left[\left(1+k_{n, s} k_{n^{\prime}, s^{\prime}}\right) L_{n^{\prime}}^{n-n^{\prime}}(\gamma)+\frac{2 n}{\epsilon_{n, s} \epsilon_{n^{\prime}, s^{\prime}}} L_{n^{\prime}-1}^{n-n^{\prime}}(\gamma)+\frac{2\left(n^{\prime}+1\right) k_{n, s} k_{n^{\prime}, s^{\prime}}}{\epsilon_{n, s} \epsilon_{n^{\prime}, s^{\prime}}} L_{n^{\prime}+1}^{n-n^{\prime}}(\gamma)\right]^{2}, \quad n \geqslant n^{\prime}
$$

Correspondingly the collisional conductivity becomes

$$
\sigma_{x x}^{\mathrm{col}, 0}=\frac{4 \beta e^{2}}{\left(L_{x} L_{y}\right)^{2}} \frac{2 \pi N_{I}}{\hbar} \sum_{\zeta, \zeta^{\prime}, q}\left|U_{q}\right|^{2}\left|J_{n n^{\prime} s s^{\prime}}(\gamma)\right|^{2} f_{n, s, k_{y}}\left(1-f_{n, s, k_{y}}\right) \delta\left(\epsilon_{n, s, k_{y}}-\epsilon_{n^{\prime}, s^{\prime}, k_{y}^{\prime}}\right) \delta_{q_{y}, k_{y}-k_{y}^{\prime}}\left[l_{B}^{4}\left(k_{y}-k_{y}^{\prime}\right)^{2}\right] .
$$

The largest contribution to the integral over $\mathbf{q}$ occurs for $q \ll k_{s}$. Accordingly we can make the approximation $U_{q}^{2} \approx U_{0}^{2}=$ $\left(2 \pi e^{2} / \varepsilon k_{s}\right)^{2}$. In addition, we have $\left(k_{y}-k_{y}^{\prime}\right)^{2}=q_{y}^{2}=q^{2} \sin ^{2} \phi$. Then the sum over $q$, converted to an integral, can be carried out in polar coordinates with the help of $\int_{0}^{\infty} \gamma e^{-\gamma}\left[L_{n}(\gamma)\right]^{2} d \gamma=2 n+1$. The dominant contribution comes from $n=n^{\prime}$ and $s=s^{\prime}$ and the sum over $q$ is $\sum_{q} q^{2} \sin \phi\left|J_{n n, s s}\right|^{2}=\left(2 L_{x} L_{y} / \pi\right) S_{n, s}$ with

$$
S_{n, s}=\left(d_{n, s}^{2} / \epsilon_{n, s}^{4}\right)\left[\left(1+k_{n, s}^{2}\right)^{2} \epsilon_{n, s}^{4}(2 n+1)+4 n^{2}(2 n-1)+4(n+1)^{2} k_{n, s}^{4}(2 n+3)\right] .
$$

When the full Eq. (23) is used we follow the procedure outlined above and obtain Eq. (26). The result for the factor $P_{n}$, appearing in it through $\Delta_{n}$, is

$$
P_{n}=\frac{d_{n-1, s} d_{n, s^{\prime}}}{\left[E_{n-1, s}-E_{n, s^{\prime}}\right] \sqrt{n}}\left[\left(1+k_{n-1, s} k_{n, s^{\prime}}\right) L_{n-1}^{1}(u)+\frac{2 n}{\epsilon_{n-1, s} \epsilon_{n, s^{\prime}}} L_{n-2}^{1}(u)+\frac{2 n k_{n-1, s} k_{n, s^{\prime}}}{\epsilon_{n-1, s} \epsilon_{n, s^{\prime}}} L_{n}^{1}(u)\right] .
$$


*mohammad.zarenia@ua.ac.be

†takis@alcor.concordia.ca

${ }^{\ddagger}$ francois.peeters@ua.ac.be

${ }^{1}$ D. Weiss, K. v. Klitzing, K. Ploog, and G. Weimann, Europhys. Lett. 8, 179 (1989); R. W. Winkler, J. P. Kotthaus, and K. Ploog, Phys. Rev. Lett. 62, 1177 (1989).

${ }^{2}$ P. Vasilopoulos and F. M. Peeters, Phys. Rev. Lett. 63, 2120 (1989); F. M. Peeters and P. Vasilopoulos, Phys. Rev. B 46, 4667 (1992).

${ }^{3}$ F. M. Peeters and P. Vasilopoulos, Phys. Rev. B 47, 1466 (1993).

${ }^{4}$ H. A. Carmona, A. K. Geim, A. Nogaret, P. C. Main, T. J. Foster, M. Henini, S. P. Beaumont, and M. G. Blamire, Phys. Rev. Lett. 74, 3009 (1995); Y. Iye, A. Endo, S. Izawa, M. Kato, and S. Katsumoto, Physica B 227, 122 (1996).

${ }^{5}$ K. S. Novoselov, D. Jiang, T. Booth, V. V. Khotkevich, S. M. Morozov, and A. K. Geim, Science 306, 6669 (2004); Y. Zhang, Y. W. Tan, H. L. Stormer, and P. Kim, Nature (London) 438, 201 (2005).

${ }^{6}$ A. Matulis and F. M. Peeters, Phys. Rev. B 75, 125429 (2007).

${ }^{7}$ R. Nasir, K. Sabeeh, and M. Tahir, Phys. Rev. B 81, 085402 (2010).

${ }^{8}$ A. Isacsson, L. M. Jonsson, J. M. Kinaret, and M. Jonson, Phys. Rev. B 77, 035423 (2008).

${ }^{9}$ T. O. Wehling, A. V. Balatsky, A. M. Tsvelik, M. I. Katsnelson, and A. I. Lichtenstein, Europhys. Lett. 84, 17003 (2008); S. Ihnatsenka, I. V. Zozoulenko, and G. Kirczenow, Phys. Rev. B 80, 155415 (2009); Z. Yu, L. Z. Sun, C. X. Zhang, and J. X. Zhong, Appl. Phys. Lett. 96, 173101 (2010); B. S. Kandemir,
Eur. Phys. J. B 78, 393 (2010); S. Costamagna, O. Hernandez, and A. Dobry, Phys. Rev. B 81, 115421 (2010).

${ }^{10}$ M. Tahir and K. Sabeeh, Phys. Rev. B 77, 195421 (2008); Y. C. Ou, J. K. Sheu, Y. H. Chiu, R. B. Chen, and M. F. Lin, ibid. 83, 195405 (2011).

${ }^{11}$ D. S. L. Abergel, V. Apalkov, J. Berashevich, K. Ziegler, and T. Chakraborty, Adv. Phys. 59, 261 (2010).

${ }^{12}$ E. V. Castro, K. S. Novoselov, S. V. Morozov, N. M. R. Peres, J. M. B. Lopes dos Santos, J. Nilsson, F. Guinea, A. K. Geim, and A. H. Castro Neto, Phys. Rev. Lett. 99, 216802 (2007).

${ }^{13}$ J. M. Pereira, F. M. Peeters, and P. Vasilopoulos, Phys. Rev. B 76, 115419 (2007).

${ }^{14}$ M. Zarenia, J. M. Pereira Jr., G. A. Farias, and F. M. Peeters, Phys. Rev. B 84, 125451 (2011).

${ }^{15}$ S. V. Morozov, K. S. Novoselov, M. I. Katsnelson, F. Schedin, D. C. Elias, J. A. Jaszczak, and A. K. Geim, Phys. Rev. Lett. 100, 016602 (2008); E. V. Castro, H. Ochoa, M. I. Katsnelson, R. V. Gorbachev, D. C. Elias, K. S. Novoselov, A. K. Geim, and F. Guinea, ibid. 105, 266601 (2010).

${ }^{16}$ P. M. Krstajić and P. Vasilopoulos, Phys. Rev. B 83, 075427 (2011).

${ }^{17}$ T. Shen, Y. Q. Wu, M. A. Capano, L. P. Rokhinson, L. W. Engel, and P. D. Ye, Appl. Phys. Lett. 93, 122102 (2008); G. Li, A. Luican, J. M. B. Lopes dos Santos, A. H. Castro Neto, A. Reina, J. Kong, and E. Y. Andrei, Nat. Phys. 6, 109 (2010).

${ }^{18}$ M. Nakamura, L. Hirasawa, and K. I. Imura, Phys. Rev. B 78, 033403 (2009). 